\title{
Penicillin and cephalosporin cross-reactivity: role of side chain and synthetic cefadroxil epitopes
}

\author{
Gador Bogas ${ }^{1,2+}$, Cristobalina Mayorga ${ }^{1,2,3 \dagger}$, Ángela Martín-Serrano 1,3 , Rubén Fernández-Santamaría', \\ Isabel M. Jiménez-Sánchez ${ }^{1,3}$, Adriana Ariza', Esther Barrionuevo 1,2, Teresa Posadas 1,2, María Salas 1,2, \\ Tahía Diana Fernández ${ }^{1,4}$, María José Torres ${ }^{1,2,3,5^{*}+}$ and María Isabel Montañez ${ }^{1,3+}$ (B)
}

\begin{abstract}
Background: Analysis of cross-reactivity is necessary for prescribing safe cephalosporins for penicillin allergic patients. Amoxicillin (AX) is the betalactam most often involved in immediate hypersensitivity reactions (IHRs), and cefadroxil (CX) the most likely cephalosporin to cross-react with AX, since they share the same R1 side chain, unlike cefuroxime (CO), with a structurally different $\mathrm{R} 1$. We aimed to analyse cross-reactivity with $C X$ and $C O$ in patients with confirmed IHRs to $A X$, including slgE recognition to $A X, C X, C O$, and novel synthetic determinants of $C X$.

Methods: Fifty-four patients with confirmed IHRs to AX based on skin test (ST) and/or drug provocation test (DPT) were included. Serum slgE to AX and benzylpenicillin was determined by Radioallergosorbent test (RAST). Two potential determinants of CX, involving intact or modified R1 structure, with open betalactam ring, were synthesised and slgE evaluated by RAST inhibition assay.

Results: Tolerance to CX (Group A) was observed in 64.8\% cases and cross-reactivity in 35.2\% cases (Group B). Crossreactivity with CO was only found in 1.8\% cases from Group B. ST to CX showed a negative predictive value of $94.6 \%$. RAST inhibition assays showed higher recognition to CX as well as to both synthetic determinants (66\% of positive cases) in Group B.
\end{abstract}

Conclusions: Cross-reactivity with CX in AX allergic patients is 35\%, being ST not enough for prediction. R1, although critical for recognition, is not the unique factor. The synthetic determinants of CX, 1-(HOPhG-Ser-Bu) and 2-(pyrazinone) are promising tools for determining in vitro cross-reactivity to CX in AX allergic patients.

Keywords: Amoxicillin, Betalactam, Cephalosporin, Cross-reactivity, Drug allergy, Antigenic determinant, Specific lgE

\section{Background}

Betalactams (BLs) are the drugs most frequently involved in immediate (IgE-mediated) hypersensitivity reactions (IHRs) [1-3], which could be explained by

\footnotetext{
*Correspondence: mjtorresj@uma.es

${ }^{\dagger}$ Gador Bogas and Cristobalina Mayorga, María José Torres and María Isabel Montañez contributed equally to this work

${ }^{1}$ Allergy Research Group, Instituto de Investigación Biomédica de Málaga-IBIMA, Hospital Civil, 29009 Málaga, Spain

Full list of author information is available at the end of the article
}

their ability to act as haptens due to their high chemical reactivity against proteins $[4,5]$. BL chemical structure is formed by a 4-membered ring (the so-called BL ring) that in penicillins is fused to a 5 -membered thiazolidine ring, and in cephalosporins to a 6-membered dihydrothiazine ring (Fig. 1). These drugs have a side chain (R1) bound to the $\mathrm{BL}$ ring; besides, cephalosporins have a second side chain (R2) bound to the dihydrothiazine ring, whose chemical structures distinguish the different compounds [6,7].

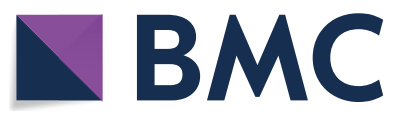

(c) The Author(s) 2020. This article is licensed under a Creative Commons Attribution 4.0 International License, which permits use, sharing, adaptation, distribution and reproduction in any medium or format, as long as you give appropriate credit to the original author(s) and the source, provide a link to the Creative Commons licence, and indicate if changes were made. The images or other third party material in this article are included in the article's Creative Commons licence, unless indicated otherwise in a credit line to the material. If material is not included in the article's Creative Commons licence and your intended use is not permitted by statutory regulation or exceeds the permitted use, you will need to obtain permission directly from the copyright holder. To view a copy of this licence, visit http://creativeco mmons.org/licenses/by/4.0/. The Creative Commons Public Domain Dedication waiver (http://creativecommons.org/publicdomain/ zero/1.0/) applies to the data made available in this article, unless otherwise stated in a credit line to the data. 


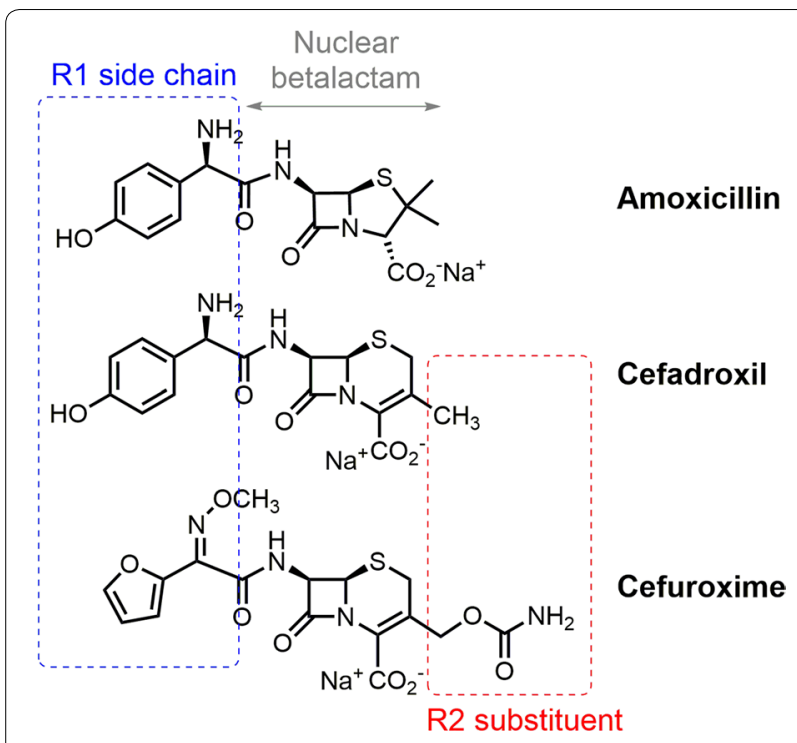

Fig. 1 Chemical structures of betalactam antibiotics involved in the study: amoxicillin (AX), cefadroxil (CX), and cefuroxime (CO); with indication of the different parts of the structures

Penicillins are the most consumed antibiotics in Europe, representing $37 \%$ of total consumption, followed by cephalosporins with a $15 \%$ of total antibiotic consumption [8]. Among them, amoxicillin (AX) is the most consumed and the most often involved in IHRs to BLs followed by cephalosporins [3, 9] which include the following: cefuroxime $(\mathrm{CO})$, ceftriaxone, cefatrizin, cefaclor, and cefadroxil (CX) [10, 11], with different percentage of cross-reactivity between them [6], highly related to their chemical structure [12-14]. Crossreactivity rate with cephalosporins in penicillin-allergic patients with IgE-mediated reactions ranges from $0 \%$ to almost $40 \%$ depending on the chemical structure of the BL involved [15-22], specifically on similarity in the $\mathrm{R} 1$ side chain $[23,24]$. In this context, $\mathrm{AX}$, which shares the same amino R1 side chain with CX (Fig. 1), could have a high cross reactivity [19-21]. Conversely, $\mathrm{CO}$, with a different $\mathrm{R} 1$ side chain, has shown tolerance in patients with IHRs to penicillins [16-19] and, more recently, similar results have been found with cefazolin and ceftibuten $[22,25]$.

Cross-reactivity has important clinical implications, especially for searching safe alternative for further treatments, and an accurate diagnosis based on skin testing (ST) is recommended, being the role of drug provocation tests (DPT) controversial $[3,9,26]$. In vitro evaluation of cross-reactivity to BLs, mainly based on immunoassays, is limited by the difficulty for studying the structure of cephalosporin-protein conjugates [27]. Although several reports have addressed this issue [28-30], the antigenic determinants of cephalosporins are currently not wellknown [31].

To our knowledge, structure-activity relationship (SAR) studies have been the unique successful approach for investigating cephalosporin epitopes [28-30, 32]. In this context, we have elucidated precise epitope structures through synthesis and immunologic evaluation of well-defined structures proposed as antigenic determinants for cephalosporins with different R1, bearing different functionalities at the C- 6 of the cephalosporin (methyl, hydroxymethyl, aldehyde, mercaptomethyl) and without involvement of the remaining dihydrothiazine ring $[29,30]$. Moreover, we have identified a novel synthetic pyrazinone structure as an antigenic determinant of cefaclor [28], formed after reaction of the amino group in the R1 with the likely aldehyde functionality at C- 6 of the original cephalosporin [28, 32]. CX is another aminocephalosporin that could follow the same fragmentation and reactivity pathways as cefaclor [32].

In this study we have evaluated the in vivo degree of cross-reactivity with $\mathrm{CX}$ and $\mathrm{CO}$ in patients with confirmed IHR to AX and the immunological recognition of AX and these cephalosporins by serum specific IgE (sIgE). The ultimate aim of this study was to evaluate if synthetic structures, proposed as potential antigenic determinants mimicking the fragment of $\mathrm{CX}$, which would remain coupled to the protein, can help get insight into the structure responsible for $\mathrm{CX}$ allergies and, therefore, study cross-reactivities between AX and CX.

\section{Methods \\ Patients}

The studied group was obtained from the Regional University Hospital of Málaga Drug Allergy Database. This prospective cohort includes all patients with confirmed drug allergy from 1984 to 2019 after an allergological workup including clinical history, ST, and DPT.

Patients with IHR to AX (allergic to the whole penicillin group or AX selective reactors with good tolerance to penicillin $\mathrm{V}(\mathrm{PV})$ ) were diagnosed following the European Academy of Allergy and Clinical Immunology (EAACI) recommendations $[9,33]$. Tolerance to $\mathrm{CX}$ and $\mathrm{CO}$ was evaluated and, based on CX tolerance, patients were classified into: Group A with tolerance (demonstrated by negative ST and DPT) and Group B with cross-reactivity (demonstrated by positive ST or DPT) (Fig. 2).

\section{Skin test}

Skin prick (SPT) and, if negative, intradermal tests (IDT) were performed as described [9, 33], using benzylpenicilloyl-poly-L-lysine (PPL, DAP, Diater, Leganés, Spain) at $1.07 \cdot 10^{-2} \mathrm{M}$, minor determinant mixture (MDM: benzylpenicillin, benzylpenicilloate, 


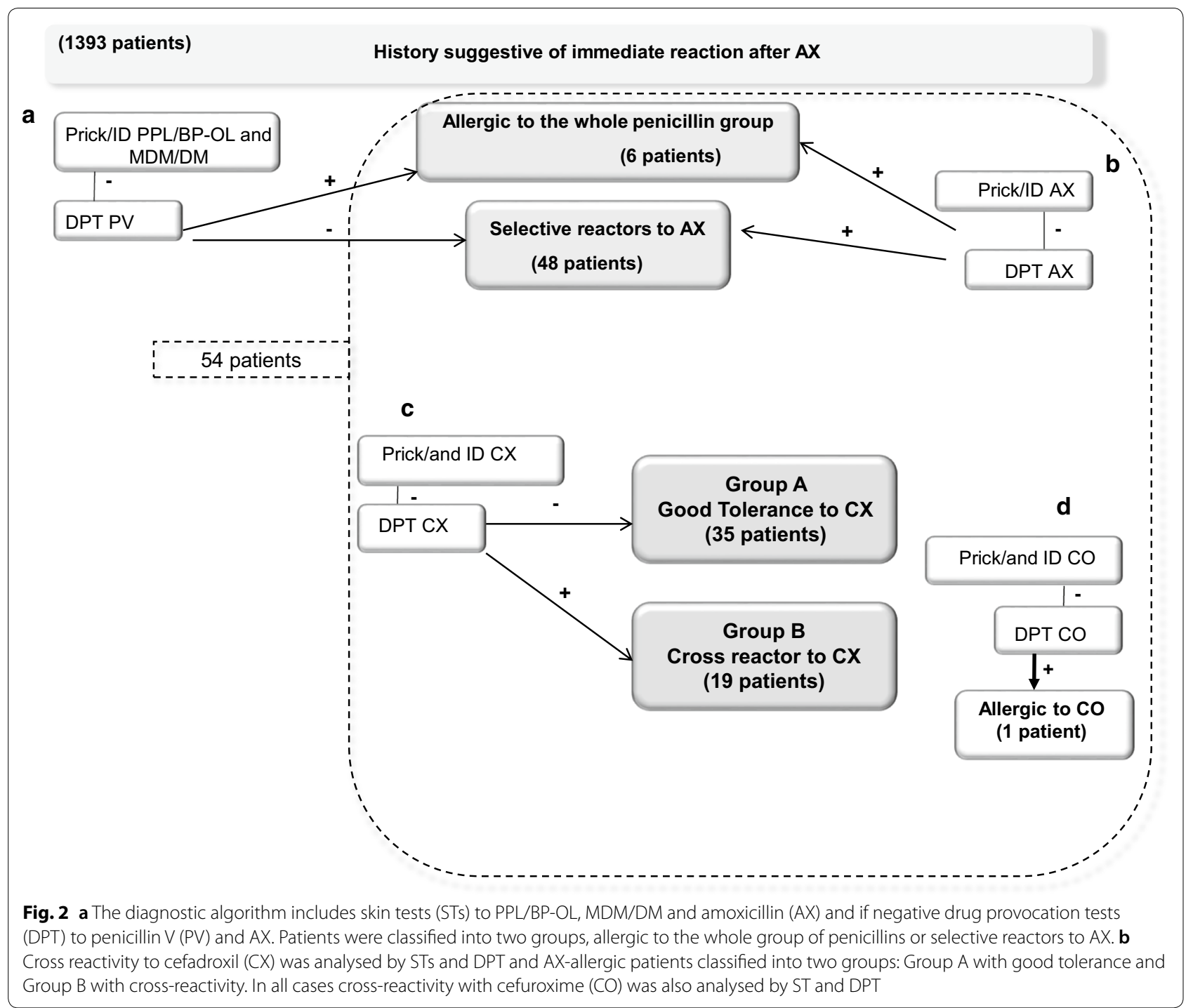

and benzylpenilloate) at $1.5 \mathrm{M}$ and $\mathrm{AX}$ (Diater laboratories, Madrid, Spain); CX (Lilly SA, Madrid) and CO (GlaxoSmithKline S.A, Madrid) all at $20 \mathrm{mg} / \mathrm{mL}$. Since May 2011 DAP composition has changed and includes the major determinant benzylpenicilloyl-octa-L-lysine (BP-OL) at $0.04 \mathrm{mg} / \mathrm{mL}$, equivalent to $8.64 \cdot 10^{-5} \mathrm{M}$ concentration of the benzylpenicilloyl (BPO) moiety, and the minor determinant (MD) at $0.5 \mathrm{mg} / \mathrm{mL}$, equivalent to $1.5 \cdot 10^{-3} \mathrm{M}$ concentration of sodium benzylpenilloate. Cephalosporin reagents were prepared according to Romano [19, 34].

Readings were done after $20 \mathrm{~min}$ and considered positive: (i) In SPT, if a wheal larger than $3 \mathrm{~mm}$ surrounded by erythema appeared, with a negative response to the control saline; (ii) In IDT, if the increase in diameter of the wheal area marked initially was greater than $3 \mathrm{~mm}$ surrounded by erythema. Positive data expressed as the mean diameter recorded by measuring the largest and the smallest diameters at right angles to each other [35].

\section{Drug provocation test}

In subjects with negative ST to PPL/BP-OL and MDM/ $\mathrm{MD}$, oral DPT with PV was performed at incremental dose $(50,100,100,150 \mathrm{mg})$ each 40 -min until reaching the total cumulative dose (TCD) of $400 \mathrm{mg}$, followed by a 2 day therapeutic course of PV of $400 \mathrm{mg} / 8$-h at home [33]. If DPT with PV and ST to AX was negative, oral DPT with AX was performed (50, 100, 150, $200 \mathrm{mg}$ ) until TCD of $500 \mathrm{mg}$, followed by a 2 day therapeutic course of AX $500 \mathrm{mg} / 8$-h at home. For cross-reactivity analysis, if ST was negative, CX was orally administered $(50,100$, 
150, $200 \mathrm{mg}$ ) until TCD of $500 \mathrm{mg}$, followed by a 2 day therapeutic course of CX $500 \mathrm{mg} / 8$-h. Finally, CO was administered following this procedure.

Patients were carefully monitored during DPT and for $2 \mathrm{~h}$ after the last dose, complete equipment for cardiopulmonary resuscitation was available [36].

\section{In vitro slgE determination by radioallergosorbent test (RAST)}

It was done using $\mathrm{BP}$ and $\mathrm{AX}$ conjugated to Poly-L-Lysine (PLL) (Sigma, St. Louis, MO) resulting in BPO-PLL and AXO-PLL in the solid phase, as described [37, 38], and radiolabeled anti-IgE antibody (kindly provided by
Thermo Fisher Scientific and radiolabelled in our laboratory) [28]. Samples were considered positive if they were higher than $2.5 \%$ of label uptake, which was the mean $+2 \mathrm{SD}$ of a negative control group.

\section{Synthesis of chemical structures}

The molecule 1 (HOPhG-Ser-Bu) (Fig. 3a) was synthesised as described [30].

The molecule 2 (pyrazinone) (Fig. 3a) was synthesised following the Ugi/Desprotect/Cyclize strategy (Fig. 3b) [39], adapting protocols from cefaclor pyrazinone synthesis [28]. The synthetic methodology and characterisation

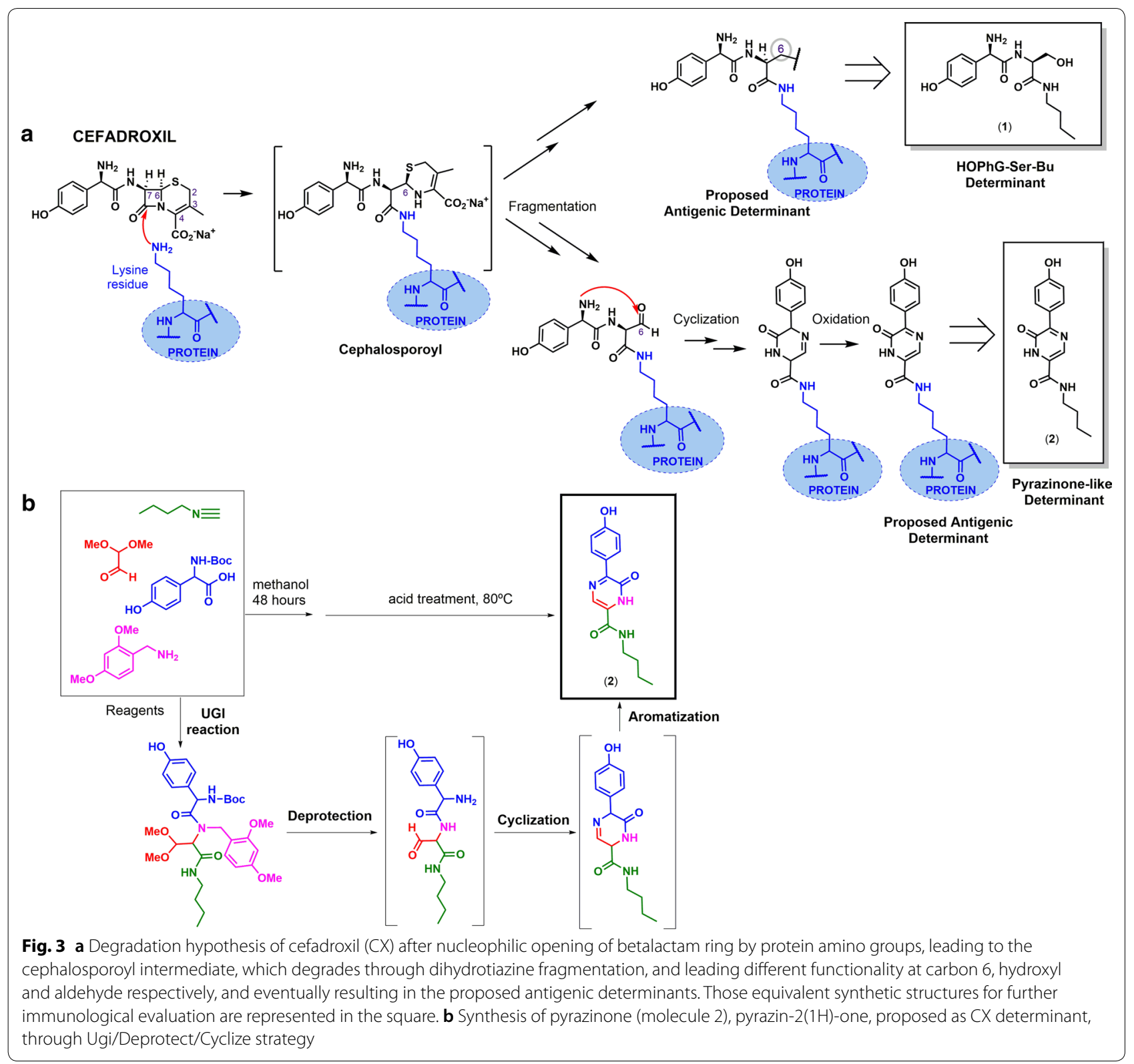


of the pure compound can be found in this article's Additional file 1.

\section{RAST inhibition assay}

This was done as described [38], incubating sera from patients with RAST values higher than $7 \%$ with different BLs (AX, CX, and CO) and the synthetic determinants of CX (1 and 2) in two ten-fold decreasing concentrations (100 mM and $10 \mathrm{mM}$ ) for $18 \mathrm{~h}$ at room temperature. After this, the AXO-PLL disc was added, followed by the previous described RAST procedure. The results were expressed as percentage inhibition with respect to the non-inhibited serum. Comparison of the inhibition capacity of the different inhibitors was made at $50 \%$ inhibition.

\section{Statistical analysis}

Description of quantitative variable included the median, mean, standard deviation (SD), and interquartile range (IR). Differences in percentage between the groups were compared by Chi-square analysis, numeric demographic data by Student $t$ test. Comparisons for variables without a normal distribution were performed by the MannWhitney test for non-related samples and by Friedman test for related samples. All statistical analyses were done using the software package GraphPad PRISM v7. A $\mathrm{P}<0.05$ was considered statistically significant.

\section{Results}

From 1393 patients with confirmed BL hypersensitivity evaluated from 1984 to 2019 , 994 subjects were confirmed with IHRS to AX, from which we randomly selected 54 patients, whose cross-reactivity to CX and $\mathrm{CO}$ was evaluated and flow-charts analysed (Fig. 2). The mean age was $41.7 \pm 12.04$ years; $35(64.8 \%)$ were males; $51(94.4 \%)$ had 1 episode and $3(5,6 \%)$ two; in $32(56.1 \%)$ episodes AX-CLV was the culprit and in 25 (43.9\%) AX. The main symptoms were anaphylaxis in 40 (70.2\%) cases, urticaria in 13 (22.8\%), and anaphylactic shock in $4(7 \%)$. The mean time interval between drug administration and development of symptoms was $26.1 \pm 19.2 \mathrm{~min}$ and between last reaction and study $132.4 \pm 131.4$ days (Table 1).

\section{Allergological work-up}

Fifty $(92.6 \%)$ patients were diagnosed by ST and 4 (7.4\%) by DPT (Tables 2 and 3). Regarding ST, 2 (4\%) cases were positive to PPL/BP-OL (both by IDT), 4 (8\%) to MDM/ MD (all by IDT), and to AX 27 (54\%) by SPT and 23 (46\%) by IDT (Table 2). Moreover, P37 and P39 developed immediate generalised pruritus and wheals 20 and 30 min respectively after ST with AX.
P1, P5, P19, and P26 were diagnosed by DPT, with 2 cases developing urticaria, 1 anaphylaxis, and 1 generalised pruritus and erythema after $\mathrm{AX}$ administration (Table 3). P8 developed anaphylaxis after PV administration. Based on ST and DPT, patients were diagnosed as selective reactors to AX $(\mathrm{N}=48,88.9 \%)$ or allergic to the whole group of penicillins $(\mathrm{N}=6,11.6 \%)$.

In all cases, ST with CX was done, with 37 (68.5\%) cases negative and $17(31.5 \%)$ positive $(6(35.3)$ by SPT and $11(64.7 \%)$ by IDT) (Table 2). From the 37 cases with negative ST to CX, DPT was done with this cephalosporin, being positive in 2 (5.4\%) (Table 3). P37 developed urticaria in trunk $50 \mathrm{~min}$ after $150 \mathrm{mg}$ of CX and needed antihistamines and corticosteroids, and P53 developed anaphylaxis $30 \mathrm{~min}$ after $50 \mathrm{mg}$ of CX and needed epinephrine.

\section{Tolerance to cefadroxil happens in $65 \%$ of AX allergic patients}

Based on CX study, 35 (64.8\%) cases showed tolerance (Group A) and 19 (35.2\%) cross-reactivity (Group B). Comparisons of the clinical characteristics of both groups showed no differences regarding type of the original reaction to penicillins, time interval between drug administration and symptom development, or time between last reaction and study. Comparisons of ST to AX showed that in Group A, 12 (34.3\%) cases were positive by SPT, 19 (54.3\%) by IDT and $4(11.4 \%)$ negative whereas in Group B 15 (78.9\%) cases were positive by SPT, $4(21.1 \%)$ by IDT, and $0(0 \%)$ negative, being differences statistically significant $(\mathrm{p}=0.006)$.

The allergological study to CO showed negative STs in all cases (Table 2) and tolerance was also confirmed in all cases by DPT (Table 3) but only P34 (Group B) developed urticaria in trunk and arms 25 min after $50 \mathrm{mg}$ of CO. Symptoms resolved $2 \mathrm{~h}$ after antihistamine administration. Therefore, cross-reactivity with $\mathrm{CO}$ was $1.8 \%$, although a concomitant sensitisation rather than a crossreactivity could be hypothesized.

\section{Significant differences of recognition are only found at the lower concentration of cefadroxil}

The analysis of sIgE results indicated that the mean value of RAST to BPO-PLL and AXO-PLL was $1.12 \pm 3.65$ and $6.8 \pm 9.4$ respectively, with 4 out of $48(8.3 \%)$ cases positive to BPO-PLL and 24 out of 48 (50\%) to AXO-PLL (Table 2). Comparisons between groups A and B showed higher differences, although not discriminating, in terms of mean levels to AXO-PLL and the percentage of positive cases (76.5\% vs 35.5\%; ) for AXO-PLL in Group B $(\mathrm{p}=0.038$ and $\mathrm{p}=0.007$, respectively).

To study CX specific recognition, we performed RAST inhibition assays on 6 cases from each group (Fig. 4a). 
Table 1 Demographic and clinical data of patients included in the study

\begin{tabular}{|c|c|c|c|c|c|c|c|c|}
\hline Pat & Group & Sex & Age & Drug & Epi & Reaction & IDR (min) & IRS (d) \\
\hline 1 & A & M & 55 & $A X-C L V$ & 1 & Anaphylaxis & 60 & 180 \\
\hline 2 & B & M & 44 & $A X-C L V$ & 1 & Anaphylaxis & 20 & 60 \\
\hline 3 & A & M & 46 & $A X$ & 1 & Anaphylaxis & 30 & 30 \\
\hline 4 & B & M & 55 & $A X$ & 1 & Urticaria & 30 & 300 \\
\hline 5 & A & M & 62 & $A X-C L V$ & 1 & Anaphylaxis & 60 & 90 \\
\hline 6 & B & $\mathrm{F}$ & 45 & $A X-C L V$ & 1 & Anaphylaxis & 10 & 30 \\
\hline 7 & B & M & 24 & $A X$ & 1 & Anaphylaxis & 10 & 30 \\
\hline 8 & B & $\mathrm{F}$ & 45 & $A X$ & 1 & Anaphylaxis & 20 & 90 \\
\hline 9 & A & M & 47 & $A X$ & 2 & $\begin{array}{l}\text { Urticaria } \\
\text { Anaphylaxis }\end{array}$ & $\begin{array}{l}40 \\
10\end{array}$ & $\begin{array}{l}477 \\
365\end{array}$ \\
\hline 10 & A & $\mathrm{F}$ & 46 & AX-CLV & 1 & Anaphylaxis & 30 & 120 \\
\hline 11 & A & M & 43 & AX-CLV & 1 & Anaphylaxis & 45 & 60 \\
\hline 12 & B & $\mathrm{F}$ & 40 & AX/AX-CLV & 2 & $\begin{array}{l}\text { Urticaria } \\
\text { Anaphylaxis }\end{array}$ & $\begin{array}{r}30 \\
5\end{array}$ & $\begin{array}{l}112 \\
109\end{array}$ \\
\hline 13 & A & F & 16 & $A X-C L V$ & 1 & Urticaria & 60 & 30 \\
\hline 14 & A & M & 27 & AX-CLV & 1 & Anaphylactic shock & 5 & 21 \\
\hline 15 & A & M & 66 & $A X$ & 1 & Anaphylaxis & 60 & 365 \\
\hline 16 & B & M & 44 & $A X$ & 1 & Anaphylaxis & 10 & 365 \\
\hline 17 & A & $\mathrm{F}$ & 50 & AX-CLV & 1 & Anaphylaxis & 15 & 30 \\
\hline 18 & A & M & 44 & $A X-C L V$ & 1 & Anaphylaxis & 10 & 280 \\
\hline 19 & A & M & 25 & AX-CLV & 1 & Urticaria & 60 & 120 \\
\hline 20 & B & $\mathrm{F}$ & 33 & AX-CLV & 1 & Anaphylaxis & 15 & 6 \\
\hline 21 & B & $\mathrm{F}$ & 36 & AX-CLV & 1 & Anaphylaxis & 30 & 60 \\
\hline 22 & A & M & 30 & $A X$ & 1 & Anaphylaxis & 5 & 20 \\
\hline 23 & B & M & 45 & $A X-C L V$ & 1 & Anaphylactic shock & 5 & 30 \\
\hline 24 & A & M & 30 & AX-CLV & 1 & Anaphylaxis & 30 & 210 \\
\hline 25 & B & M & 57 & AX-CLV & 1 & Anaphylaxis & 5 & 60 \\
\hline 26 & A & M & 49 & $A X$ & 1 & Anaphylaxis & 10 & 120 \\
\hline 27 & A & $\mathrm{F}$ & 39 & $A X$ & 1 & Urticaria & 40 & 40 \\
\hline 28 & A & $\mathrm{F}$ & 39 & $A X-C L V$ & 1 & Urticaria & 60 & 95 \\
\hline 29 & A & $\mathrm{F}$ & 21 & $A X-C L V$ & 1 & Anaphylaxis & 15 & 230 \\
\hline 30 & A & M & 46 & AX-CLV & 1 & Anaphylaxis & 2 & 137 \\
\hline 31 & B & M & 49 & $A X$ & 1 & Anaphylaxis & 5 & 180 \\
\hline 32 & B & $\mathrm{F}$ & 37 & AX-CLV & 1 & Anaphylaxis & 20 & 30 \\
\hline 33 & A & $\mathrm{F}$ & 57 & $A X$ & 1 & Anaphylaxis & 30 & 90 \\
\hline 34 & B & M & 26 & $A X$ & 1 & Anaphylaxis & 20 & 10 \\
\hline 35 & B & M & 48 & $A X-C L V$ & 1 & Anaphylaxis & 60 & 146 \\
\hline 36 & A & M & 23 & AX-CLV & 1 & Anaphylaxis & 50 & 60 \\
\hline 37 & B & $\mathrm{F}$ & 42 & $A X$ & 1 & Anaphylaxis & 5 & 120 \\
\hline 38 & B & M & 57 & $A X-C L V$ & 1 & Anaphylaxis & 30 & 28 \\
\hline 39 & B & M & 30 & $A X-C L V$ & 1 & Urticaria & 10 & 730 \\
\hline 40 & A & $\mathrm{F}$ & 37 & AX-CLV & 1 & Anaphylaxis & 5 & 30 \\
\hline 41 & A & $\mathrm{F}$ & 14 & $A X-C L V$ & 1 & Anaphylaxis & 10 & 90 \\
\hline 42 & A & M & 44 & $A X$ & 1 & Urticaria & 30 & 120 \\
\hline 43 & A & M & 28 & $A X$ & 2 & $\begin{array}{l}\text { Anaphylaxis } \\
\text { Anaphylaxis }\end{array}$ & $\begin{array}{l}20 \\
25\end{array}$ & $\begin{array}{l}230 \\
180\end{array}$ \\
\hline 44 & A & M & 63 & $A X-C L V$ & 1 & Anaphylaxis & 10 & 30 \\
\hline 45 & A & M & 51 & $A X$ & 1 & Urticaria & 60 & 30 \\
\hline 46 & A & M & 35 & $A X$ & 1 & Urticaria & 30 & 90 \\
\hline 47 & A & M & 32 & $A X$ & 1 & Anaphylaxis & 45 & 180 \\
\hline
\end{tabular}


Table 1 (continued)

\begin{tabular}{|c|c|c|c|c|c|c|c|c|}
\hline Pat & Group & Sex & Age & Drug & Epi & Reaction & IDR (min) & IRS (d) \\
\hline 48 & A & M & 53 & $A X$ & 1 & Anaphylaxis & 30 & 90 \\
\hline 49 & A & M & 44 & $A X$ & 1 & Urticaria & 45 & 120 \\
\hline 50 & A & $\mathrm{F}$ & 38 & AX-CLV & 1 & Anaphylaxis & 10 & 30 \\
\hline 51 & A & M & 54 & $A X-C L V$ & 1 & Anaphylactic shock & 5 & 90 \\
\hline 52 & A & $\mathrm{F}$ & 62 & $A X-C L V$ & 1 & Urticaria & 60 & 210 \\
\hline 53 & B & M & 49 & $A X-C L V$ & 1 & Anaphylaxis & 30 & 120 \\
\hline 54 & A & $\mathrm{F}$ & 44 & $A X$ & 1 & Anaphylactic shock & 5 & 240 \\
\hline
\end{tabular}

Patients were classified into Group A (Good tolerance to cefadroxil) or Group B (Cross-reactivity with cefadroxil)

Pat, Patients; M, Male; F, Female; AX-CLV, Amoxicillin-clavulanic; AX, Amoxicillin; Epi, Number of episodes; IDR (min), Interval drug administration and development of symptoms in minutes; IRS (d), Interval last reaction and allergological study in days

As inhibitors, we included $\mathrm{AX}, \mathrm{CX}$, and $\mathrm{CO}$ at two concentrations, 10 and $100 \mathrm{mM}$ (Fig. 4b). Results with AX showed, as expected, a high percentage of inhibition at both concentrations in all cases. Regarding CX, the percentage of inhibition was above $50 \%$ in most of patients at $100 \mathrm{mM}, 5$ out of 6 patients in each group, similarly to levels obtained with AX. However, these percentages decrease at $10 \mathrm{mM}$, being lower than those observed with AX especially Group A (Fig. 4a). In fact, comparison analysis of the percentage of inhibition between groups only shows significant differences for $\mathrm{CX}$ at $10 \mathrm{mM}(\mathrm{p}=0.034)$ (Fig. 4c). Only one case (P38, Group B) showed a percentage above $50 \%$ with $\mathrm{CO}$.

\section{Synthetic determinants of cefadroxil showed better slgE recognition in Group B}

The design of the two synthetic determinants of CX was based on our degradation hypothesis of the aminocephalosporin-protein conjugate, using butylamine as a model nucleophile emulating protein lysine (Fig. 3a). After covalent protein conjugation through $\mathrm{BL}$ ring, the dihydrothiazine ring is unstable and could degrade producing structures in which carbon 6 presents different functionalities. Two relevant candidates, according to previous immunological recognition results [30], are structures bearing hydroxyl and aldehyde functionality in carbon 6 . In the case of hydroxyl functionality, it would generate the molecule $\mathbf{1}$ as determinant; whereas the aldehyde functionality can react with the amino group of R1 side chain generating the pyrazinone $\mathbf{2}$ as a novel determinant. The synthesis of the molecule 2 was achieved following the Ugi/Deprotect/Cyclize strategy (Fig. 3b) [39]. First, starting reagents (an isocyanide, a protected amine, a protected aldehyde, and a $\mathrm{N}$-protected aminoacid) were assembled by following the one-pot Ugi four-component reaction to produce the Ugi adduct. The latter acidmediated-cleavage of the protected groups may result in the amino-functionalised aldehyde intermediate that cyclises, through intramolecular imine formation, and aromatises affording target pyrazinone (2). This method allowed the straightforward synthesis of 2, for which other procedures resulted unsuccessful. Compounds $\mathbf{1}$ and $\mathbf{2}$ were purified and well-characterised, allowing the immunological recognition study of precise chemical structures.

RAST inhibition assays were performed using CX and the two synthetic structures (1 and 2), as inhibitors (Fig. 5b), in two cases from Group A and 6 from Group B. There was no inhibition with these structures in Group A (Fig. 5a). Higher percentages of inhibition were observed in Group B, being greater than $50 \%$ in 4 out of 6 cases at $100 \mathrm{mM}$, in which similar levels of inhibition to those obtained with CX were observed (Fig. 5a). However, significant lower percentage of inhibition with these synthetic structures was observed performing the RAST inhibition at $10 \mathrm{mM}$ ( $\mathrm{p}=0.0022$ for both) (Fig. 5c).

\section{Discussion}

BLs are the most widely used antibiotics and the drugs most frequently involved in IHR [1] in adults and children [40-42]. All BL compounds can potentially induce a specific immunological response and, due to their wide prescription, BL allergy is nowadays a worldwide health issue with relevant implications [43-45]. One of the main issues is establishing the risk of developing an allergic reaction to cephalosporins prescribed in patients previously diagnosed of penicillin IHR, with different unsolved questions like if this risk can be predicted by ST and/or DPT, or the role of the chemical structure, specifically the side chain, in this recognition [10, 46-49]. The main difficulty is that, despite efforts [28-30], the antigenic determinants of cephalosporins are unknown [31]. In this study we have found that, for predicting cross-reactivity, ST is not enough and, R1 side chain, although critical for recognition, is not the unique factor. Moreover, the use of chemical tools for SAR study is a promising approach 
Table 2 Skin tests and RAST results in patients from Group A (Good tolerance to cefadroxil) and Group B (Cross-reactivity with cefadroxil)

\begin{tabular}{|c|c|c|c|c|c|c|c|c|}
\hline \multirow[t]{2}{*}{ Pat } & \multirow[t]{2}{*}{ Group } & \multicolumn{5}{|l|}{ Skin test } & \multirow{2}{*}{$\begin{array}{l}\text { RAST } \\
\text { BPO-PLL }\end{array}$} & \multirow[t]{2}{*}{ AX-PLL } \\
\hline & & PPL/BP-OL & MDM/MD & $A X$ & $C X$ & $\mathrm{CO}$ & & \\
\hline 1 & A & Neg & Neg & Neg & Neg & Neg & ND & ND \\
\hline 2 & B & Neg & Neg & $\mathrm{SPT}+(5 \times 5)$ & $\mathrm{ID}+(2 \times 2)$ & Neg & 0 & 3.42 \\
\hline 3 & A & Neg & Neg & $\mathrm{SPT}+(5 \times 5)$ & Neg & Neg & 0.15 & 3.24 \\
\hline 4 & B & Neg & Neg & $\mathrm{SPT}+(4 \times 5)$ & $\mathrm{SPT}+(4 \times 4)$ & Neg & 0 & 0 \\
\hline 5 & A & Neg & Neg & Neg & Neg & Neg & 0 & 0 \\
\hline 6 & B & Neg & Neg & $\mathrm{SPT}+(5 \times 5)$ & IDT $+(1 \times 1)$ & Neg & ND & ND \\
\hline 7 & B & Neg & Neg & $\mathrm{SPT}+(6 \times 6)$ & $\mathrm{SPT}+(3 \times 3)$ & Neg & 0.34 & 14.59 \\
\hline 8 & B & Neg & Neg & $\mathrm{SPT}+(8 \times 1)$ & $\mathrm{SPT}+(5 \times 5)$ & Neg & 0 & 3.51 \\
\hline 9 & A & Neg & Neg & $\mathrm{SPT}+(5 \times 5)$ & $\mathrm{Neg}$ & Neg & 0 & 0 \\
\hline 10 & A & Neg & Neg & $\mathrm{IDT}+(3 \times 2)$ & Neg & Neg & 0 & 0 \\
\hline 11 & A & Neg & Neg & $\mathrm{IDT}+(2 \times 2)$ & Neg & Neg & 0 & 0 \\
\hline 12 & B & Neg & Neg & $\mathrm{IDT}+(3 \times 3)$ & IDT + (2×2) & Neg & 0.2 & 0.46 \\
\hline 13 & A & Neg & Neg & $\mathrm{IDT}+(4 \times 4)$ & Neg & Neg & ND & ND \\
\hline 14 & A & Neg & Neg & $\mathrm{SPT}+(3 \times 3)$ & Neg & Neg & 0 & 0 \\
\hline 15 & A & Neg & Neg & $\mathrm{IDT}+(2 \times 3)$ & Neg & Neg & ND & ND \\
\hline 16 & B & Neg & Neg & $\mathrm{SPT}+(4 \times 5)$ & $\mathrm{IDT}+(2 \times 2)$ & Neg & 1.72 & 11.71 \\
\hline 17 & A & Neg & Neg & $\mathrm{IDT}+(2 \times 3)$ & Neg & Neg & 0 & 0 \\
\hline 18 & A & Neg & Neg & $\mathrm{IDT}+(3 \times 4)$ & Neg & Neg & 0 & 0 \\
\hline 19 & A & Neg & Neg & Neg & Neg & Neg & ND & ND \\
\hline 20 & B & Neg & Neg & $\mathrm{IDT}+(2 \times 2)$ & IDT $+(2 \times 1)$ & Neg & 0 & 3.2 \\
\hline 21 & B & Neg & Neg & $\mathrm{SPT}+(5 \times 6)$ & IDT $+(1 \times 2)$ & Neg & ND & ND \\
\hline 22 & A & $\mathrm{IDT}+(2 \times 1)$ & IDT+(1×1) & $\mathrm{SPT}+(3 \times 3)$ & Neg & Neg & 3.2 & 6.79 \\
\hline 23 & B & Neg & IDT+(2×2) & $\mathrm{SPT}+(6 \times 6)$ & $\mathrm{SPT}+(3 \times 3)$ & Neg & 23.54 & 29.83 \\
\hline 24 & A & Neg & Neg & $\mathrm{SPT}+(2 \times 3)$ & Neg & Neg & 0 & 3.03 \\
\hline 25 & B & Neg & Neg & $\mathrm{SPT}+(5 \times 6)$ & $\mathrm{IDT}+(2 \times 2)$ & Neg & 1.22 & 7.55 \\
\hline 26 & A & Neg & Neg & Neg & Neg & Neg & 0 & 0 \\
\hline 27 & A & Neg & Neg & $\mathrm{IDT}+(3 \times 3)$ & IDT $+(2 \times 2)$ & Neg & 0.2 & 0.46 \\
\hline 28 & A & Neg & Neg & $\mathrm{IDT}+(3 \times 4)$ & Neg & Neg & 2.15 & 8.32 \\
\hline 29 & A & Neg & Neg & $\mathrm{IDT}+(4 \times 4)$ & Neg & Neg & 0.09 & 0.54 \\
\hline 30 & A & Neg & Neg & $\mathrm{SPT}+(5 \times 6)$ & Neg & Neg & 0 & 1.14 \\
\hline 31 & B & Neg & Neg & $\mathrm{SPT}+(5 \times 5)$ & $\mathrm{SPT}+(2+3)$ & Neg & 0 & 22.54 \\
\hline 32 & B & Neg & Neg & $\mathrm{SPT}+(3 \times 3)$ & $\mathrm{IDT}+(2 \times 3)$ & Neg & 0 & 15.56 \\
\hline 33 & A & Neg & Neg & $\mathrm{SPT}+(4 \times 5)$ & Neg & Neg & 1.28 & 2.31 \\
\hline 34 & B & Neg & Neg & $\mathrm{SPT}+(6 \times 6)$ & IDT $+(2 \times 3)$ & Neg & 1.87 & 3.36 \\
\hline 35 & B & Neg & $\mathrm{IDT}+(2 \times 2)$ & $\mathrm{SPT}+(7 \times 8)$ & $\mathrm{SPT}+(3 \times 4)$ & Neg & 6.15 & 26.09 \\
\hline 36 & A & Neg & Neg & $\mathrm{IDT}+(2 \times 2)$ & Neg & Neg & 0 & 25.56 \\
\hline 37 & B & Neg & $\mathrm{Neg}$ & $\mathrm{IDT}+(1 \times 2)$ & Neg & Neg & 0.37 & 0.13 \\
\hline 38 & B & Neg & Neg & $\mathrm{SPT}+(5 \times 6)$ & IDT + (3×4) & Neg & 1.65 & 21.8 \\
\hline 39 & B & Neg & Neg & $\mathrm{IDT}+(2 \times 2)$ & Neg & Neg & 0 & 0.92 \\
\hline 40 & A & Neg & Neg & $\mathrm{SPT}+(2 \times 3)$ & Neg & Neg & 0 & 0.7 \\
\hline 41 & A & Neg & Neg & $\mathrm{IDT}+(5 \times 6)$ & Neg & Neg & 0 & 1.93 \\
\hline 42 & A & Neg & Neg & $\mathrm{IDT}+(2 \times 2)$ & Neg & Neg & 0 & 0.37 \\
\hline 43 & A & Neg & Neg & $\mathrm{IDT}+(3 \times 3)$ & Neg & Neg & 0 & 1.24 \\
\hline 44 & A & Neg & Neg & $\mathrm{IDT}+(4 \times 5)$ & Neg & Neg & 0.23 & 0 \\
\hline 45 & A & Neg & Neg & $\mathrm{IDT}+(2 \times 2)$ & Neg & Neg & 0 & 23.2 \\
\hline 46 & A & Neg & $\mathrm{Neg}$ & $\mathrm{SPT}+(4 \times 4)$ & Neg & Neg & 0 & 0 \\
\hline 47 & A & Neg & $\mathrm{ID}+(2 \times 2)$ & $\mathrm{SPT}+(5 \times 6)$ & Neg & Neg & 0.84 & 1.12 \\
\hline
\end{tabular}


Table 2 (continued)

\begin{tabular}{|c|c|c|c|c|c|c|c|c|}
\hline \multirow[t]{2}{*}{ Pat } & \multirow[t]{2}{*}{ Group } & \multicolumn{5}{|l|}{ Skin test } & \multirow{2}{*}{$\begin{array}{l}\text { RAST } \\
\text { BPO-PLL }\end{array}$} & \multirow[t]{2}{*}{ AX-PLL } \\
\hline & & PPL/BP-OL & MDM/MD & $A X$ & $C X$ & $\mathrm{CO}$ & & \\
\hline 48 & A & $\mathrm{ID}+(2 \times 2)$ & Neg & $\mathrm{SPT}+(4 \times 5)$ & Neg & Neg & 8.13 & 13.87 \\
\hline 49 & A & Neg & Neg & $\mathrm{IDT}+(2 \times 2)$ & Neg & Neg & 0 & 0.14 \\
\hline 50 & A & Neg & Neg & $\mathrm{SPT}+(5 \times 4)$ & Neg & Neg & 0.54 & 0.41 \\
\hline 51 & A & Neg & Neg & $\mathrm{IDT}+(5 \times 7)$ & Neg & Neg & 0 & 14.68 \\
\hline 52 & A & Neg & Neg & $\mathrm{IDT}+(3 \times 4)$ & Neg & Neg & 0 & 7.93 \\
\hline 53 & B & Neg & Neg & $\mathrm{SPT}+(4 \times 5)$ & Neg & Neg & 0 & 16.84 \\
\hline 54 & A & Neg & $\mathrm{Neg}$ & $\mathrm{IDT}+(3 \times 2)$ & Neg & Neg & 0.06 & 33.02 \\
\hline
\end{tabular}

Pat, Patients; PPL/BPO-OL, Benzylpenicilloyl-poly-L-lysine/benzylpenicilloyl-octa-L-lysine; MDM/MD, Minor determinant mixture/minor determinant; AX, Amoxicillin; CX, Cefadroxil; CO, Cefuroxime; BPO-PLL, Benzylpenicilloyl-poly-L-lysine; AXO-PLL, Amoxicilloyl-poly-L-lysine; SPT, Skin prick test; IDT, Intradermal test; Neg, Negative; ND, Not done

for elucidating the chemical structures involved in these IHRs.

In this study we have found that the cross-reactivity of IHRs to AX with CX, a cephalosporin with the same R1, was $35 \%$ and with $\mathrm{CO}$, cephalosporin with different R1, figures decrease to $1.8 \%$. Results with $\mathrm{CX}$ are in agreement with those by Romano [19] reporting that 39.3\% of patients with IHR to penicillins had positive tests for cephalosporins, with $37.7 \%$ positive to aminocephalosporins, including CX and/or cefamandole. These results are similar to our previous data, with $38 \%$ of cross-reactivity between AX and CX using DPT [21]. Relevantly, we did not detect differences in cross-reactivity to $\mathrm{CX}$ among those selective to AX compared to those reacting to the whole group, confirming that R1 is not the only factor influencing cross-reactivity. Regarding $\mathrm{CO}$ tolerance, all patients had negative ST and only one had a positive DPT, showing a high degree of CO tolerance, in line with previous data [16-19,25].

Comparisons of SPT results to AX between Group A and $\mathrm{B}$ (cross-reactivity to $\mathrm{CX}$ ) showed a higher percentage of positivity (78.9 vs $34.3 \%$ ) in the group tolerant to CX (Group A). These results agree with those by Romano [19] estimating an odds ratio of ST positivity to ampicillin for cross-reacting to at least one cephalosporin of 2.5 (CI, 1.4-4.5). Moreover, the analysis of the sIgE results showed significantly higher levels and positivity to AXOPLL in Group B. This seems to indicate that patients that cross-react with cephalosporins have a high degree of reactivity, taking into account that the two cases that developed systemic symptoms after ST with penicillins belong to Group B and that patients reacted to small amount of CX (50 and $150 \mathrm{mg}$ ) and CO (50 mg) in DPT.

Regarding the role of ST for predicting cross-reactivity, a positive cephalosporin ST in patients allergic to penicillins may indicate not only cross-reactivity but also concomitant sensitivity. Of note, P34 with cross-reaction to
$\mathrm{CO}$ also reacted to $\mathrm{CX}$. Whether this patient has crossreacting or co-existing antibodies was something we cannot clarify in the present study as the RAST level was not enough for performing RAST inhibition with both drugs. However, cross-reactivity is more probable since this patient had not been previously treated with cefuroxime or any cephalosporin. This percentage is in agreement with previous data [18] that found $2.9 \%$ of cross-reactivity with $\mathrm{CO}$ in patients with prior histories involving only a penicillin. If a negative cephalosporin ST predicts good tolerance is controversial [50]. Different studies showed that patients with a well-established IgE-mediated allergy to penicillin and with ST negative to cephalosporins tolerate cephalosporins [15-17]. However, others demonstrated that less than $3 \%$ of cases can have a DPT positive with cephalosporin despite having negative ST [18, 19]. In this study 2 out of 37 patients (5.4\%) with ST negative to $C X$ and 1 out of 54 patients (1.8\%) with ST negative to $\mathrm{CO}$ had a positive DPT to CX and CO respectively, indicating a negative predictive value (NPV) of $94.6 \%$ for CX and $98.1 \%$ for CO. That means that although NPV are high, a negative ST does not mean tolerance even if R1 are different.

Our immunological study by RAST inhibition assays agrees with previous results on cross-reactivity between penicillins and cephalosporins, showing that AX presented a better recognition, followed by CX [21]. Data showed a discriminating capacity of the test between Group A and B using lower drug concentrations, $10 \mathrm{mM}$, observing a significantly lower recognition of $\mathrm{CX}$ in patients with good tolerance to CX (Group A).

Regardless of this discriminative capacity, these data indicate that, although important for IgE recognition, the $\mathrm{R} 1$ is not per se the only structure involved in the immunological response, as structural modifications or some fragments of the nuclear structure may be involved in the antigenic determinant. In penicillins, the penicilloyl 
Table 3 Drug provocation test results in patients from Group A (Good tolerance to cefadroxil) and Group B (Cross-reactivity with cefadroxil)

\begin{tabular}{|c|c|c|c|c|c|}
\hline Pat & Group & Drug & Reaction & IDR (min) & $\mathrm{TCD}$ (mg) \\
\hline \multirow[t]{2}{*}{1} & A & $A X$ & Urticaria & 160 & 500 \\
\hline & & PV/CX/CO & Good tolerance & - & - \\
\hline 2 & B & $\mathrm{PV} / \mathrm{CO}$ & Good tolerance & - & - \\
\hline 3 & A & PV/CX/CO & Good tolerance & - & - \\
\hline 4 & B & $\mathrm{PV} / \mathrm{CO}$ & Good tolerance & - & - \\
\hline \multirow[t]{2}{*}{5} & A & $A X$ & Anaphylaxis & 15 & 50 \\
\hline & & PV/CX/CO & Good tolerance & - & - \\
\hline 6 & B & $\mathrm{PV} / \mathrm{CO}$ & Good tolerance & - & - \\
\hline 7 & B & $\mathrm{PV} / \mathrm{CO}$ & Good tolerance & - & - \\
\hline \multirow[t]{2}{*}{8} & B & PV & Anaphylaxis & 45 & 150 \\
\hline & & $\mathrm{CO}$ & Good tolerance & - & - \\
\hline 9 & A & $\mathrm{PV} / \mathrm{CX} / \mathrm{CO}$ & Good tolerance & - & - \\
\hline 10 & $A$ & PV/CX/CO & Good tolerance & - & - \\
\hline 11 & $A$ & PV/CX/CO & Good tolerance & - & - \\
\hline 12 & B & $\mathrm{PV} / \mathrm{CO}$ & Good tolerance & - & - \\
\hline 13 & A & PV/CX/CO & Good tolerance & - & - \\
\hline 14 & A & PV/CX/CO & Good tolerance & - & - \\
\hline 15 & A & PV/CX/CO & Good tolerance & - & - \\
\hline 16 & B & $\mathrm{PV} / \mathrm{CO}$ & Good tolerance & - & - \\
\hline 17 & A & PV/CX/CO & Good tolerance & - & - \\
\hline 18 & A & PV/CX/CO & Good tolerance & - & - \\
\hline \multirow[t]{2}{*}{19} & $A$ & $A X$ & Urticaria & 140 & 500 \\
\hline & & PV/CX/CO & Good tolerance & - & - \\
\hline 20 & B & $\mathrm{PV} / \mathrm{CO}$ & Good tolerance & - & - \\
\hline 21 & B & $\mathrm{PV} / \mathrm{CO}$ & Good tolerance & - & - \\
\hline 22 & A & $\mathrm{CX} / \mathrm{CO}$ & Good tolerance & - & - \\
\hline 23 & B & $\mathrm{CO}$ & Good tolerance & - & - \\
\hline 24 & A & PV/CX/CO & Good tolerance & - & - \\
\hline 25 & $\mathrm{~B}$ & $\mathrm{PV} / \mathrm{CO}$ & Good tolerance & - & - \\
\hline \multirow[t]{2}{*}{26} & A & $A X$ & $\begin{array}{l}\text { Generalized } \\
\text { pruritus and } \\
\text { erythema }\end{array}$ & 60 & 150 \\
\hline & & PV/CX/CO & Good tolerance & - & - \\
\hline 27 & A & $\mathrm{PV} / \mathrm{CO}$ & Good tolerance & - & - \\
\hline 28 & A & PV/CX/CO & Good tolerance & - & - \\
\hline 29 & $A$ & PV/CX/CO & Good tolerance & - & - \\
\hline 30 & $A$ & PV/CX/CO & Good tolerance & - & - \\
\hline 31 & B & $\mathrm{PV} / \mathrm{CO}$ & Good tolerance & - & - \\
\hline 32 & B & $\mathrm{PV} / \mathrm{CO}$ & Good tolerance & - & - \\
\hline 33 & A & PV/CX/CO & Good tolerance & - & - \\
\hline \multirow[t]{2}{*}{34} & B & PV & Good tolerance & - & - \\
\hline & & $\mathrm{CO}$ & Urticaria & 25 & 50 \\
\hline 35 & $\mathrm{~B}$ & $\mathrm{CO}$ & Good tolerance & - & - \\
\hline 36 & A & PV/CX/CO & Good tolerance & - & - \\
\hline \multirow[t]{2}{*}{37} & B & $\mathrm{PV} / \mathrm{CO}$ & Good tolerance & - & - \\
\hline & & $C X$ & Urticaria & 50 & 150 \\
\hline 38 & B & $\mathrm{PV} / \mathrm{CO}$ & Good tolerance & - & - \\
\hline 39 & B & $\mathrm{PV} / \mathrm{CX} / \mathrm{CO}$ & Good tolerance & - & - \\
\hline
\end{tabular}

Table 3 (continued)

\begin{tabular}{llllll}
\hline Pat & Group & Drug & Reaction & IDR (min) & TCD (mg) \\
\hline 40 & A & PV/CX/CO & Good tolerance & - & - \\
41 & A & PV/CX/CO & Good tolerance & - & - \\
42 & A & PV/CX/CO & Good tolerance & - & - \\
43 & A & PV/CX/CO & Good tolerance & - & - \\
44 & A & PV/CX/CO & Good tolerance & - & - \\
45 & A & PV/CX/CO & Good tolerance & - & - \\
46 & A & PV/CX/CO & Good tolerance & - & - \\
47 & A & CX/CO & Good tolerance & - & - \\
48 & A & CX/CO & Good tolerance & - & - \\
49 & A & PV/CX/CO & Good tolerance & - & - \\
50 & A & PV/CX/CO & Good tolerance & - & - \\
51 & A & PV/CX/CO & Good tolerance & - & - \\
52 & A & PV/CX/CO & Good tolerance & - & - \\
53 & B & PV/CO & Good tolerance & - & - \\
& & CX & Anaphylaxis & 30 & 50 \\
54 & A & PV/CX/CO & Good tolerance & - & - \\
\hline
\end{tabular}

Time Interval and Total cumulative dose drug administration and the development of symptoms

Pat, Patients; AX, Amoxicillin; PV, Penicillin V; CX, Cefadroxil; CO, Cefuroxime; IDR ( $\mathrm{min})$, Interval between drug administration and development of symptoms in minutes; $T C D$, Total cumulative dose in $\mathrm{mg}$

structure formed after protein conjugation is stable and, therefore, the thiazolidine ring could also play a role in the antigenic determinant [51-53]. On the contrary, the equivalent cephalosporyl structure is unstable, thus the R2 substituent is expulsed $[54,55]$ and the dihydrothiazine ring suffers different fragmentations, producing a complex mixture in which structures are difficult to elucidate $[29,31]$. We have addressed this issue, by using chemical tools, for performing SAR studies in which precisely defined structures, consisting on the R1 side chain coupled to the open BL ring with the carbon 6 of the original drug represented by a methyl group, were recognised by sIgE from patients with IHR to the cephalosporin containing either the same R1 or one structurally similar [29]. Further SAR studies involved similar synthetic determinants but with different functionalisation in such carbon 6, finding that hydroxymethyl and aldehyde functionality, compared with methyl group, increased recognition [30]. Based on these results, synthetic determinants of $\mathrm{CX}$, involving the whole intact R1 or a modified R1 side chain, have been immunologically evaluated, showing higher-recognition by sIgE from patients cross-reactive to CX (Group B).

The structure 1 (HPhG-Ser-Bu), consisting on the $\mathrm{R} 1$ side chain of $\mathrm{CX}$ and open $\mathrm{BL}$ ring with hydroxymethyl functionality at carbon 6 [30], was not previously evaluated with sIgE to aminocephalosporins. These determinants containing the intact corresponding 


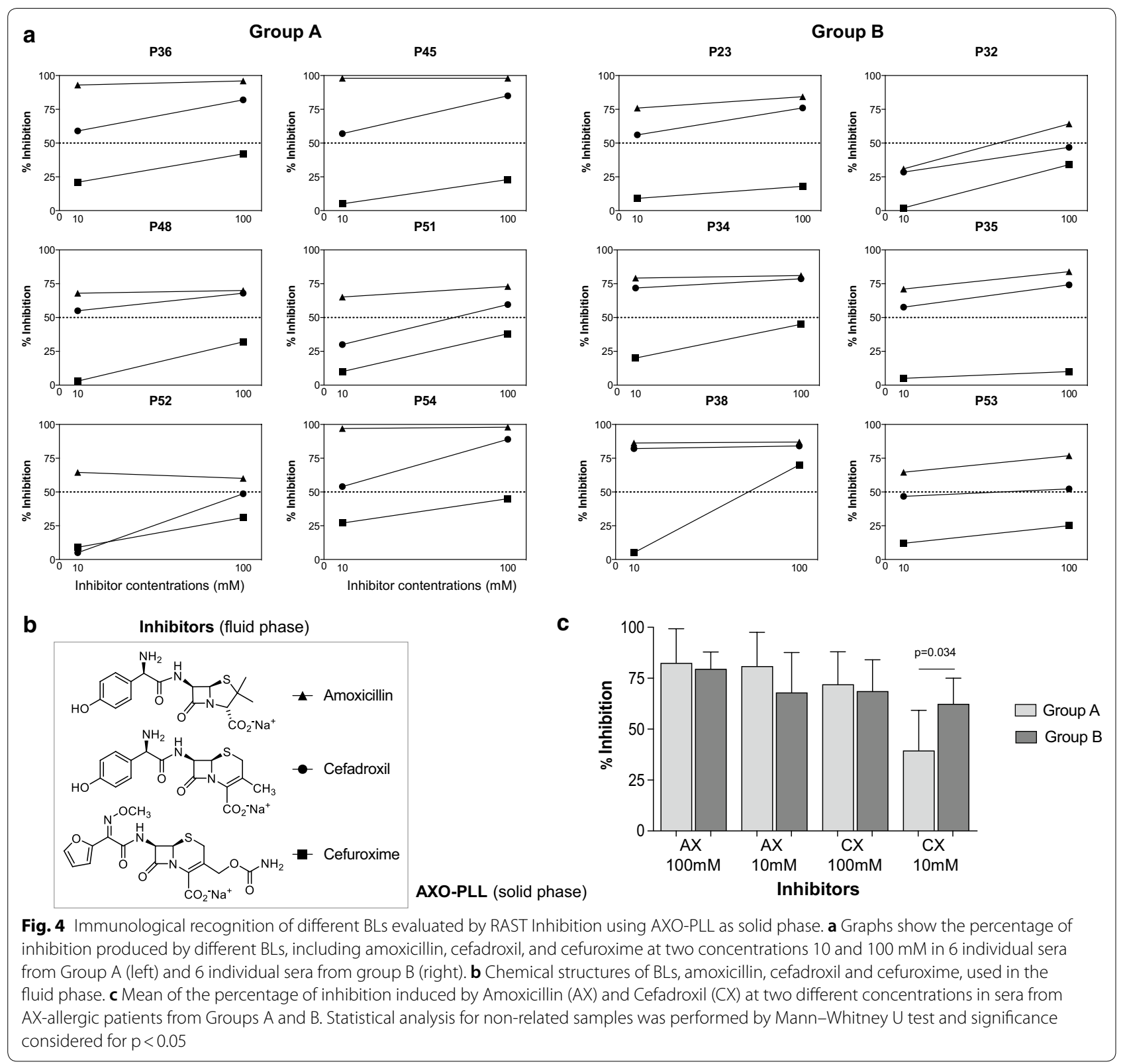

aminocephalosporins R1 have been immunologically evaluated in a recent study with cefaclor-allergic patients (12\% of positive cases) [28], and in the present study with AX-and/or CX-allergic patients (66\% of positive cases at the maximum concentration), showing different extent of recognition depending on $\mathrm{R} 1$.

The pyrazinone 2 has been synthesised and immunologically evaluated in this study for the first time. Its structure derives from intramolecular reaction between the $\mathrm{R} 1$ amino group and the aldehyde at carbon 6 . Inhibition results in six cases of Group B show that the pyrazinone 2 , at $100 \mathrm{mM}$ concentration, is recognised in $66 \%$ of cases, in agreement with IgE recognition observed for pyrazinones derived from cefaclor, with $63 \%$ of positive cases for the equivalent pyrazinone to that described here [28], and $60 \%$ of patients for an equivalent analog developed by Venemalm [32].

These synthetic determinants (1 and $\mathbf{2})$ were not recognised by the two selected patients with tolerance to CX (Group A). Importantly, greater differences in recognition 


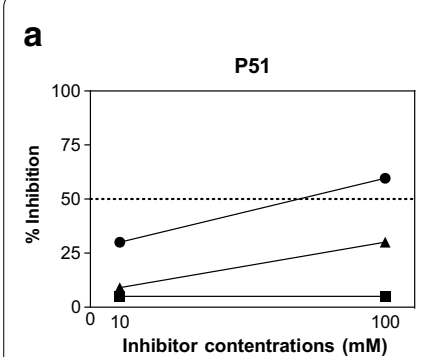

\section{Group A}

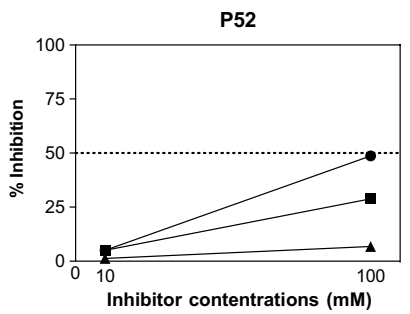

Group B

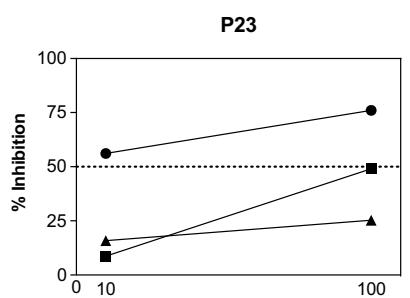

P34

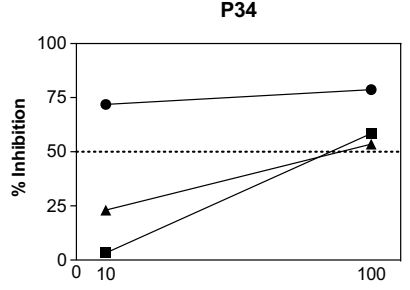

P38

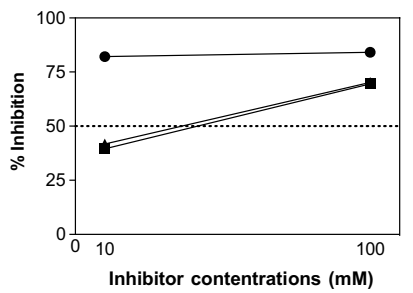

P32

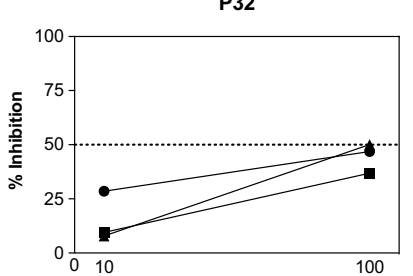

P35

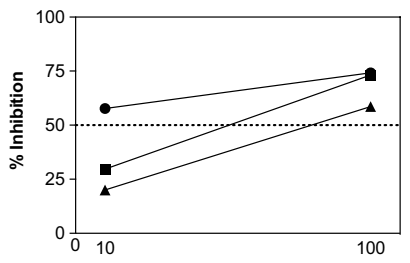

P53

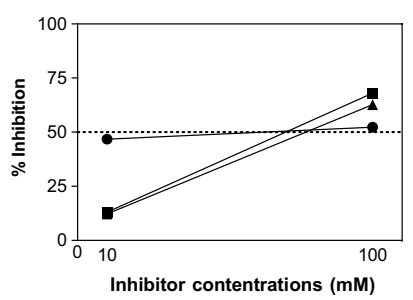

b

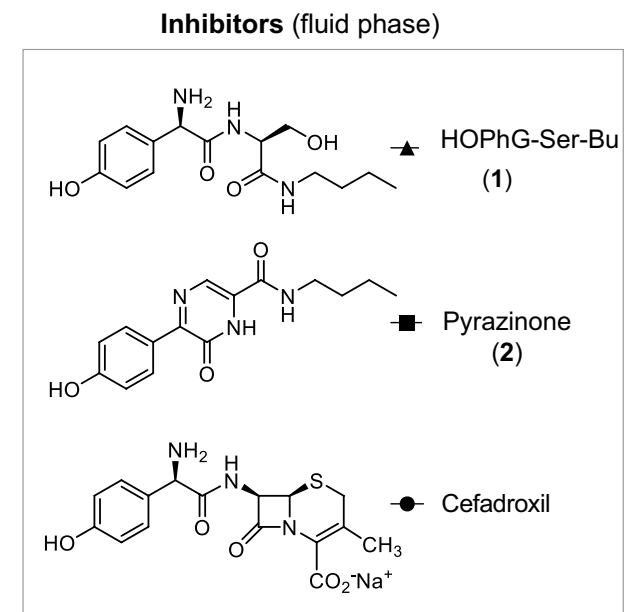

AXO-PLL (solid phase)

C

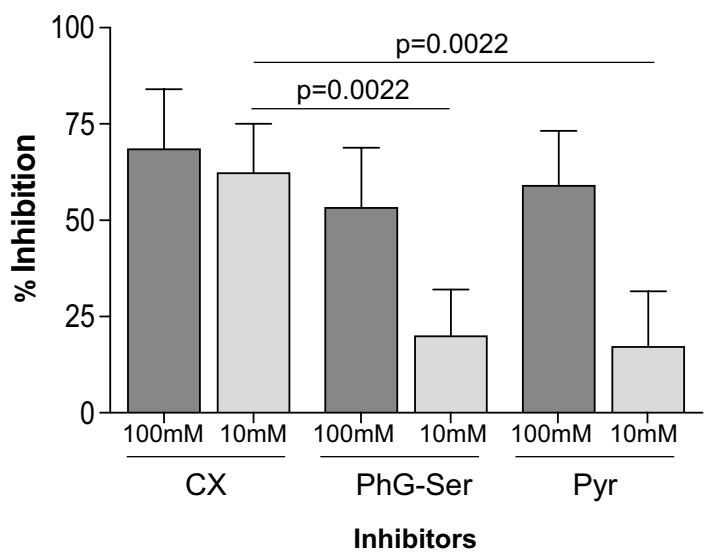

Fig. 5 Immunological evaluation results with cefadroxil (CX) and its synthetic antigenic determinants. a RAST inhibition assays performed with sera from AX-allergic patients, 2 from Group A and 6 patients from Group B. b Chemical structure of inhibitors, synthetic compounds HOPhG-Ser-Bu (1), Pyrazinone (2) and native CX, and solid phase (amoxicilloyl-poly-L-Lysine, AXO-PLL). c Bars represent mean of percentage of inhibition for the three inhibitors at 100 and $10 \mathrm{mM}$ concentrations in Group B. Statistical comparisons among related samples by Friedman test for related samples being significant with $p$ values $<0.05$

between $\mathrm{CX}$ and the synthetic structures were observed in Group A than in Group B, using the higher concentration.

One could think that AX presents the amino group in $\mathrm{R} 1$ for the formation of additional determinants, as diketopiperazine, considered as a minor determinant of AX [56]. However, it did not show sIgE recognition in previous studies [57], which is consistent with its lack of reactivity with proteins [56].

\section{Conclusions}

We have confirmed that cross-reactivity between penicillin and cephalosporins occurs when the R1 side chain is identical as previously reported, and that negative ST is not enough for predicting tolerance, being DPT necessary. The primary determinant of immunochemical recognition of aminocephalosporins rested, with the structure of the R1, intact (molecule $\mathbf{1}$ ) or in its cyclised form as pyrazinone (molecule 2 ), although other parts of the molecule (excluding R2 substituents and most of the dihydrothiazine) are necessary for the formation of 
the antigenic determinant. These structures represent useful and safe alternatives for determining in vitro cross-reactivity to CX in AX-allergic patients. We think that other determinants, involving different patterns of recognition, could also participate in CX-allergic reactions; and more research is needed in this regard.

\section{Supplementary Information}

The online version contains supplementary material available at https://doi. org/10.1186/s13601-020-00368-1.

Additional file 1: Figure S1. Nuclear Magenteic Resonance (NMR) characterization of structure 2. (A) ${ }^{1} \mathrm{H}-\mathrm{NMR}\left(\mathrm{CH}_{3} \mathrm{OD}\right)$ spectrum, (B) ${ }^{13} \mathrm{C}-\mathrm{NMR}$ $\left(\mathrm{CH}_{3} \mathrm{OD}\right)$ spectrum, and $(\mathrm{C})$ heteronuclear single quantum coherence (HSQC) experiment with gradient pulse. Bidimensional NMR spectrum (left) and signal assignation (right).

\section{Abbreviations}

AX: Amoxicillin; AXO: Amoxicilloyl; BL: Betalactam; BP: Benzylpenicillin; BPO: Benzylpenicilloyl; BP-OL: Benzylpenicilloyl-octa-L-lysine; CO: Cefuroxime; CX: Cefadroxil; DPT: Drug provocation test; EAACl: European Academy of Allergy and Clinical Immunology; IDT: Intradermal tests; IHR: Immediate hypersensitivity reactions; IR: Interquartile range; MD: Minor determinant; MDM: Minor determinant mixture; NPV: Negative predictive value; PPL: Benzylpenicilloyl-poly-L-lysine; PLL: Poly-L-Lysine; PV: Penicillin V; RAST: Radioallergosorbent test; SAR: Structure-activity relationship; slgE: Specific lgE; ST: Skin testing; SD: Standard deviation; SPT: Skin prick test; TCD: Total cumulative dose.

\section{Acknowledgments}

We thank Ms Claudia Corazza for her help with the English version of the manuscript.

\section{Authors' contributions}

Concepts, Design, Definition of intellectual content-GB, CM, TDF, MIM, MJT. Literature search-GB, CM, AM-S, RF-S, TDF, AA, MIM, MJT. Clinical studies: GB, EB, TP, MS, MJT. Clinical data analysis - GB, CM, TDF, MS, MJT. Immunoassay studies and data analysis-CM, AM-S, RF-S, AA, IMJ-S, MIM. Chemical synthesis and structural elucidation-AM-S, MIM. Statistical analysis-CM, TDF, MJT. Manuscript preparation and editing - GB, CM, MIM, MJT. All authors have read and approved the final manuscript.

\section{Funding}

The present study has been supported by the Institute of Health "Carlos III" (ISCIII) of MINECO (Grants cofunded by ERDF: "Una manera de hacer Europa": Grant ns. PI12/02529, PI15/01206, CP15/00103, PI17/01237, PI18/00095, RETIC ARADYAL RD16/0006/0001, Euronanomed Program AC19/00082; Andalusian Regional Ministry of Economy and Knowledge (Grants cofunded by ERDF: "Andalucía se mueve con Europa": Grant No. CTS-06603); Andalusian Regional Ministry of Health (Grant Nos. PI-0699-2011, PI-0179-2014, PE-01722018 cofunded by ERDF); and "Premio UNICAJA a la innovación en biomedicina y salud." C.M. holds 'Nicolas Monardes' research contract by Andalusian Regional Ministry Health (Grant No. C-0044-2012 SAS2013). G.B. holds a "Juan Rodes" Grant (JR18/00054), M.I.M. holds a "Miguel Servet I"Grant (CP15/00103), and A.A. holds a "Sara Borrell" Grant (CD17/0146), all by ISCIII of MINECO (grants cofunded by European Social Fund: "El FSE invierte en futuro"). R.F.S. holds a predoctoral Grant (PE-0172-2018) cofunded by ERDF.

\section{Availability of data and materials}

All data generated or analysed during this study are included in this published article and its supplementary Additional file 1.

\section{Ethics approval and consent to participate}

The studied group was obtained from the Regional University Hospital of Málaga Drug Allergy Database. The study was approved by the institutional review board, and informed consent for all procedures was obtained from all patients.

\section{Competing interests}

The group collaborates in research grants with Diater Laboratories (Madrid, Spain). The authors declare no other relevant conflicts of interest.

\section{Author details}

${ }^{1}$ Allergy Research Group, Instituto de Investigación Biomédica de MálagaIBIMA, Hospital Civil, 29009 Málaga, Spain. ${ }^{2}$ Allergy Unit, Hospital Regional Universitario de Málaga, Hospital Civil, 29009 Málaga, Spain. ${ }^{3}$ Nanostructures for Diagnosing and Treatment of Allergic Diseases Laboratory, Andalusian Center for Nanomedicine and Biotechnology-BIONAND, Parque Tecnológico de Andalucía, 29590 Málaga, Spain. ${ }^{4}$ Departamento de Biología Celular, Genética y Fisiología, Universidad de Málaga, 29071 Málaga, Spain. ${ }^{5}$ Departamento de Medicina, Universidad de Málaga, Facultad de Medicina, 29071 Málaga, Spain.

Received: 10 October 2020 Accepted: 27 November 2020

Published online: 04 December 2020

\section{References}

1. Doña I, Barrionuevo E, Blanca-López N, Torres M, Fernandez T, Mayorga C, et al. Trends in hypersensitivity drug reactions: more drugs, more response patterns, more heterogeneity. J Investig Allergol Clin Immunol. 2014;24(3):143-53.

2. Demoly P, Adkinson NF, Brockow K, Castells M, Chiriac AM, Greenberger PA, et al. International consensus on drug allergy. Allergy. 2014;69(4):420-37.

3. Torres MJ, Celik GE, Whitaker P, Atanaskovic-Markovic M, Barbaud A, Bircher A, et al. A EAACI drug allergy interest group survey on how European allergy specialists deal with beta-lactam allergy. Allergy. 2019;74(6):1052-62.

4. Sánchez-Gómez FJ, González-Morena JM, Vida Y, Pérez-Inestrosa E, Blanca M, Torres MJ, et al. Amoxicillin haptenates intracellular proteins that can be transported in exosomes to target cells. Allergy. 2017;72:385-96.

5. Ariza A, Fernandez TD, Mayorga C, Barbero N, Martin-Serrano A, Perez-Sala D, et al. Hypersensitivity reactions to Betalactams: relevance of the hapten-protein conjugates. J Investig Allergol Clin Immunol. 2015;25(1):12-25.

6. Montañez Ml, Ariza A, Mayorga C, Fernandez T, Torres M. Cross-reactivity in betalactam allergy: alternative treatments. Current Treatment Options in Allergy. 2015;2:141-54

7. Martín-Serrano A, Barbero N, Agundez JA, Vida Y, Pérez-Inestrosa E, Montañez MI. New advances in the study of IgE drug recognition. Curr Pharm Des. 2016;22:1-14.

8. WHO report on surveillance of antibiotic consumption: 2016-2018 early implementation:: Geneva: World Health Organization. Licence: CC BY-NCSA 3.0 IGO.; 2018.

9. Romano A, Atanaskovic-Markovic M, Barbaud A, Bircher AJ, Brockow K, Caubet JC, et al. Towards a more precise diagnosis of hypersensitivity to beta-lactams - an EAACl position paper. Allergy. 2019. https://doi. org/10.1111/all.14122.

10. Zagursky RJ, Pichichero ME. Cross-reactivity in $\beta$-Lactam allergy. J Allergy Clin Immunol Pract. 2018;6(1):72-81.e1.

11. Renaudin JM, Beaudouin E, Ponvert C, Demoly P, Moneret-Vautrin DA Severe drug-induced anaphylaxis: analysis of 333 cases recorded by the Allergy Vigilance Network from 2002 to 2010. Allergy. 2013;68(7):929-37.

12. Pichler WJ. Immune pathomechanism and classification of drug hypersensitivity. Allergy. 2019;74(8):1457-71.

13. Kowalski ML, Agache I, Bavbek S, Bakirtas A, Blanca M, Bochenek G, et al. Diagnosis and management of NSAID-exacerbated Respiratory Disease (N-ERD)—a EAACI position paper. Allergy. 2019;74(1):28-39.

14. Brockow K, Ardern-Jones MR, Mockenhaupt M, Aberer W, Barbaud A, Caubet J-C, et al. EAACl position paper on how to classify cutaneous manifestations of drug hypersensitivity. Allergy. 2019;74(1):14-27.

15. Audicana M, Bernaola G, Urrutia I, Echechipia S, Gastaminza G, Muñoz $D$, et al. Allergic reactions to betalactams: studies in a group of patients allergic to penicillin and evaluation of cross-reactivity with cephalosporin. Allergy. 1994;49(2):108-13. 
16. Novalbos A, Sastre J, Cuesta J, De Las HM, Lluch-Bernal M, Bombín C, et al. Lack of allergic cross-reactivity to cephalosporins among patients allergic to penicillins. Clin Exp Allergy. 2001;31(3):438-43.

17. Romano A, Guéant-Rodriguez R-M, Viola M, Pettinato R, Guéant J-L. Crossreactivity and tolerability of cephalosporins in patients with immediate hypersensitivity to penicillins. Ann Intern Med. 2004;141(1):16-22.

18. Caimmi S, Galera C, Bousquet-Rouanet L, Arnoux B, Demoly P, Bousquet PJ. Safety of cefuroxime as an alternative in patients with a proven hypersensitivity to penicillins: a DAHD cohort survey. Int Arch Allergy Immunol. 2010;153(1):53-60.

19. Romano A, Valluzzi RL, Caruso C, Maggioletti M, Quaratino D, Gaeta F. Cross-reactivity and tolerability of cephalosporins in patients with lgEmediated hypersensitivity to penicillins. J Allergy Clin Immunol Pract. 2018;6(5):1662-72.

20. Sastre J, Quijano LD, Novalbos A, Hernandez G, Cuesta J, delas Heras $M$, et al. Clinical cross-reactivity between amoxicillin and cephadroxil in patients allergic to amoxicillin and with good tolerance of penicillin. Allergy. 1996;51(6):383-6.

21. Miranda A, Blanca M, Vega JM, Moreno F, Carmona MJ, García JJ, et al. Cross-reactivity between a penicillin and a cephalosporin with the same side chain. J Allergy Clin Immunol. 1996;98(3):671-7.

22. Romano A, Valluzzi RL, Caruso C, Zaffiro A, Quaratino D, Gaeta F. Tolerability of cefazolin and ceftibuten in patients with IgE-. J Allergy Clin Immunol Pract. 2020. https://doi.org/10.1016/j.jaip.2020.02.025.

23. Romano A, Mayorga C, Torres MJ, Artesani MC, Suau R, Sanchez F, et al. Immediate allergic reactions to cephalosporins: cross-reactivity and selective responses. J Allergy Clin Immunol. 2000;106(6):1177-83.

24. Antunez C, Blanca-Lopez N, Torres MJ, Mayorga C, Perez-Inestrosa E, Montañez MI, Fernandez T, Blanca M. Immediate allergic reactions to cephalosporins: evaluation of cross-reactivity with a panel of penicillins and cephalosporins. J Allergy Clin Immunol. 2006;117(2):404-10.

25. de Vicente J, Gamboa P, García-Lirio E, Irazabal B, Jáuregui I, Martínez MD, et al. Tolerance to cephalosporins and carbapenems in penicillin-allergic patients. J Investig Allergol Clin Immunol. 2020;30(1):75-6.

26. Mayorga C, Celik G, Rouzaire P, Whitaker P, Bonadonna P, Cernadas JR, et al. In vitro tests for drug hypersensitivity reactions. An ENDA/EAACl drug allergy interest group position paper. Allergy. 2016;71(8):1103-34.

27. Barbero N, Fernández-Santamaría R, Mayorga C, Martin-Serrano Á, Salas $M$, Bogas $G$, et al. Identification of an antigenic determinant of clavulanic acid responsible for lgE-mediated reactions. Allergy. 2019;74(8):1490-501.

28. Martín-Serrano A, Mayorga C, Barrionuevo E, Pérez N, Romano A, Moreno $E_{1}$ et al. Design of an antigenic determinant of cefaclor: chemical structure-lgE recognition relationship. J Allergy Clin Immunol. 2020;145(4):1301-4.e4.

29. Sanchez-Sancho F, Perez-Inestrosa E, Suau R, Montañez MI, Mayorga C, Torres MJ, et al. Synthesis, characterization and immunochemical evaluation of cephalosporin antigenic determinants. J Mol Recognit. 2003;16:148-56

30. Montañez Ml, Mayorga C, Torres MJ, Ariza A, Blanca M, Perez-Inestrosa E. Synthetic approach to gain insight into antigenic determinants of cephalosporins: in vitro studies of chemical structure-lgE molecular recognition relationships. Chem Res Toxicol. 2011;24(5):706-17.

31. Perez-Inestrosa E, Suau R, Montañez MI, Rodriguez R, Mayorga C, Torres MJ, et al. Cephalosporin chemical reactivity and its immunological implications. Curr Opin Allergy Clin Immunol. 2005:5(4):323-30.

32. Venemalm L. Pyrazinone conjugates as potential cephalosporin allergens. Bioorg Med Chem Lett. 2001;1 1(14):1869-70.

33. Doña I, Romano A, Torres MJ. Algorithm for betalactam allergy diagnosis. Allergy. 2019;74(9):1817-9.

34. Romano A, Guéant-Rodriguez RM, Viola M, Amoghly F, Gaeta F, Nicolas JP, et al. Diagnosing immediate reactions to cephalosporins. Clin Exp Allergy 2005;35(9):1234-42

35. Brockow K, Romano A, Blanca M, Ring J, Pichler W, Demoly P. General considerations for skin test procedures in the diagnosis of drug hypersensitivity. Allergy. 2002;57(1):45-51.

36. Aberer W, Bircher A, Romano A, Blanca M, Campi P, Fernandez J, et al. Drug provocation testing in the diagnosis of drug hypersensitivity reactions: general considerations. Allergy. 2003;58(9):854-63.

37. Fontaine C, Mayorga C, Bousquet PJ, Arnoux B, Torres MJ, Blanca M, et al. Relevance of the determination of serum-specific lgE antibodies in the diagnosis of immediate beta-lactam allergy. Allergy. 2007;62(1):47-52.
38. Antunez C, Fernandez T, Blanca-Lopez N, Torres MJ, Mayorga C, Canto $\mathrm{G}$, et al. IgE antibodies to betalactams: relationship between the triggering hapten and the specificity of the immune response. Allergy. 2006;61(8):940-6.

39. Azuaje J, El Maatougui A, Pérez-Rubio JM, Coelho A, Fernández F, Sotelo E. Multicomponent assembly of diverse pyrazin-2(1H)-one chemotypes. J Org Chem. 2013;78(9):4402-9.

40. Rubio M, Bousquet PJ, Gomes E, Romano A, Demoly P. Results of drug hypersensitivity evaluations in a large group of children and adults. Clin Exp Allergy. 2012;42(1):123-30.

41. Gomes ER, Brockow K, Kuyucu S, Saretta F, Mori F, Blanca-Lopez N, et al. Drug hypersensitivity in children: report from the pediatric task force of the EAACI Drug Allergy Interest Group. Allergy. 2016;71(2):149-61.

42. Torres MJ, Blanca M. The complex clinical picture of $\beta$-lactam hypersensitivity: penicillins, cephalosporins, monobactams, carbapenems, and clavams. Med Clin North Am. 2010;94(4):805-20.

43. Mayorga C, FernandezTD, Montañez MI, Moreno E, Torres MJ. Recent developments and highlights in drug hypersensitivity. Allergy. 2019;74(12):2368-81.

44. Stone CA Jr, Trubiano J, Coleman DT, Rukasin CRF, Phillips EJ. The challenge of de-labeling penicillin allergy. Allergy. 2020;75(2):273-88.

45. Sousa-Pinto B, Blumenthal KG, Macy E, Bavbek S, Benić MS, Alves-Correia $\mathrm{M}$, et al. Diagnostic testing for penicillin allergy: a survey of practices and cost perceptions. Allergy. 2020;75(2):436-41.

46. Romano A, Gaeta F, Arribas Poves MF, Valluzzi RL. Cross-reactivity among beta-lactams. Curr Allergy Asthma Rep. 2016;16(3):24.

47. Picard M, Robitaille G, Karam F, Daigle JM, Bédard F, Biron É, et al. Crossreactivity to cephalosporins and carbapenems in penicillin-allergic patients: two systematic reviews and meta-analyses. J Allergy Clin Immunol Pract. 2019;7(8):2722-38.e5.

48. Mirakian R, Leech SC, Krishna MT, Richter AG, Huber PA, Farooque S, et al. Management of allergy to penicillins and other beta-lactams. Clin Exp Allergy. 2015:45(2):300-27.

49. Yang M-S, Kang DY, Seo B, Park HJ, Park S-Y, Kim M-Y, et al. Incidence of cephalosporin-induced anaphylaxis and clinical efficacy of screening intradermal tests with cephalosporins: a large multicenter retrospective cohort study. Allergy. 2018;73(9):1833-41.

50. Blanca M, Romano A, Torres MJ, Férnandez J, Mayorga C, Rodriguez J, et al. Update on the evaluation of hypersensitivity reactions to betalactams. Allergy. 2009;64(2):183-93.

51. Ariza A, Garzon D, Abánades DR, de Ríos V, Vistoli G, Torres MJ, et al. Protein haptenation by amoxicillin: High resolution mass spectrometry analysis and identification of target proteins in serum. J Proteomics. 2012;77:504-20.

52. Torres MJ, Montañez MI, Ariza A, Salas M, Fernandez TD, Barbero N, et al. The role of IgE recognition in allergic reactions to amoxicillin and clavulanic acid. Clin Exp Allergy. 2016;46(2):264-74.

53. Ariza A, Mayorga C, Salas M, Doña I, Martín-Serrano Á, Pérez-Inestrosa $E$, et al. The influence of the carrier molecule on amoxicillin recognition by specific lgE in patients with immediate hypersensitivity reactions to betalactams. Sci Rep. 2016;6:35113.

54. Pratt RF, Faraci WS. Direct observation by 1H-NMR of cephalosporoate intermediates in aqueous solution during the hydrazinolysis and $\beta$-lactamasecatalized hydrolysis of cephalosporin with 3' leaving groups: kinetics and equilibria of the $3^{\prime}$ elimination reaction. J Am Chem Soc. 1986;108:5328-33.

55. Montañez Ml, Martín-Serrano A, Mayorga C, Barrionuevo E, Pérez N, Romano A, et al. Reply. J Allergy Clin Immunol. 2020.

56. Pajares MA, Zimmerman T, Sánchez-Gómez FJ, Ariza A, Torres MJ, Blanca $M$, et al. Amoxicillin inactivation by thiol-catalyzed cyclization reduces protein haptenation and antibacterial potency. Front Pharmacol. 2020;1 1:189.

57. Torres MJ, Ariza A, Fernández J, Moreno E, Laguna JJ, Montañez Ml, et al. Role of minor determinants of amoxicillin in the diagnosis of immediate allergic reactions to amoxicillin. Allergy. 2010;65(5):590-6.

\section{Publisher's Note}

Springer Nature remains neutral with regard to jurisdictional claims in published maps and institutional affiliations. 